\title{
R. Vettor \\ The metabolic actions of thyroid hormone and leptin: a mandatory interplay or not?
}

Received: 14 December 2004 / Accepted: 2 February 2005 / Published online: 12 March 2005

(C) Springer-Verlag 2005

\section{Introduction}

The report by P. Cettour-Rose and colleagues on the metabolic consequences of hypothyroidism in rats and their partial correction by central leptin infusion in this month's issue of Diabetologia [1] represents a further step in highlighting the importance of both central and peripheral interplay between thyroid hormones and leptin in the control of energy metabolism. The authors measured whole-body glucose turnover by euglycaemic-hyperinsulinaemic clamp and tissue-specific glucose utilisation in propylthiouracilinduced hypothyroid rats using the labelled 2-deoxy-D-glucose technique. Leptin was infused intracerebroventricularly (i.c.v.) after the induction of hypothyroidism in a group of rats. One of the leptin-infused hypothyroid groups was additionally infused subcutaneously with reverse T3 (rT3) to inhibit type 2 deiodinase activity. Leptin-infused rats treated with rT3 were therefore deficient in both type 1 and type 2 deiodinase activities. In this paper the authors further confirm the presence of decreased overall glucose turnover and glucose utilisation in skeletal muscle and adipose tissue of hypothyroid rats, together with a reduction in leptin and an increase in resistin mRNA expression. Leptin infusion $i$. c.v. in hypothyroid rats partially restores the impairment in glucose metabolism, and this seems to occur independently of T3 levels and resistin expression. The effect of i.c.v. leptin also resulted in increased expression of carnitine palmitoyl transferases, decreased plasma NEFA levels and reduced muscle triglyceride content. The authors conclude that increased glucose-fatty acid competition may explain the decreased insulin responsiveness in hypothyroidism, and that this phenomenon is partly alleviated by i.c.v. leptin administration.

R. Vettor $(\bowtie)$

Department of Medical and Surgical Sciences,

Clinica Medica 3, University of Padua, University Hospital,

via Ospedale 105,

35128 Padua, Italy

e-mail: roberto.vettor@unipd.it

Tel.: +39-049-8212648

Fax: +39-049-8213333
The effect of thyroid hormone on basal metabolic rate was recognised more than a century ago by A. MagnusLevy [2]. The thermogenic effect of thyroid hormone is generally coupled to increased food intake and lipogenesis, and thyrotoxic rats eat more, have increased lipogenesis, and predominantly oxidise fat for energy supply. The role of increased appetite is to maintain weight in the presence of accelerated metabolism; increased lipogenesis, on the other hand, ensures the fuel supply for heat production, thus preventing thermogenesis from depleting fat stores and body proteins. Studies in the adult rat have shown that T3 plays important roles in regulating basal oxygen consumption, fat stores, lipogenesis and lipolysis [3].

The mechanisms by which thyroid hormone stimulates food intake remain undefined. Several studies have tried to elucidate a possible relationship between leptin and thyroid dysfunction but so far no clear conclusions have been drawn. Serum leptin was found to be decreased in hyperthyroid rats, while increased or unchanged serum leptin levels were found in hypothyroid rats. In the current study, leptinaemia was lower in hypothyroid rats than in control animals [1]. Wang et al. reported that the effects of leptin in reducing food intake and body weight and in stimulating energy metabolism were not dependent on the presence of thyroid hormone, and suggested that leptin and thyroid hormones might share some common downstream action sites and could act additively, although independently, to enhance energy metabolism [4]. Thyroid hormones might have physiological inhibitory effects on serum leptin since the inverse relationship between leptin and thyroid hormones was maintained over a wide range of thyroid hormone levels, from severe hypothyroidism to hyperthyroidism [4, 5]. Moreover, changes in body fat content are likely to contribute to the decrease in leptin as seen in chronically hyperthyroid rats, and this reduction may mediate the increase in food intake.

There is increasing evidence that central leptin could influence the hypothalamo-pituitary-thyroid axis [6-9], although the series of events underlying this phenomenon is not fully understood. Leptin could enhance the activity of brown adipose tissue (BAT) monodeiodinase type II (D2) 
via activation of the sympathetic nervous system, increasing plasma T3 levels. Such an increase would favour the activity of hepatic monodeiodinase type I (D1), given the high sensitivity of this enzyme to T3 [10]. Leptin action could therefore bear on D2 activity in BAT initially, and subsequently on D1 activity in the liver, ultimately contributing to increased plasma T3 levels.

Thyroid hormones regulate multiple cellular processes and functions in every type of tissue. They modulate glucose metabolism by direct actions, which include the synthesis of key enzymes and alterations in other regulatory hormones, such as insulin, glucagon and catecholamines. In rats, thyroid hormone deficiency has been linked with a striking decrease in the responsiveness of glucose uptake and glycogen synthesis to insulin in muscle [11]. In contrast, treatment of hypothyroid rats with T3 directly stimulates basal and insulin-mediated glucose uptake in rat skeletal muscle [12]. The mechanism for this induction was shown to be due to an increase in GLUT4 mRNA and protein expression $[12,13]$.

Hypothyroidism also results in the decreased expression of mitochondrial oxidative enzymes, and experimental hyperthyroidism causes an increase in the same enzymes. Several of the proteins that increase in response to excess thyroid hormones also increase in response to chronic chemical activation of AMP-activated protein kinase (AMPK) using 5-amino-4-imidazolecarboxamide riboside [14]. The expression of AMPK and of acetyl coenzyme A carboxylase (ACC) in skeletal muscle is influenced by the thyroid state. Muscle ACC activity, protein expression and phosphorylation are increased with thyroid hormone treatment. It is possible that increases in basal AMPK activity would result in chronic enhancement of glucose uptake, GLUT4/ hexokinase/mitochondrial enzyme expression and fatty acid oxidation. All these processes are enhanced in response to hyperthyroidism. It has been observed that hyperthyroid rats have markedly lower malonyl-CoA levels in their muscles compared with the propylthiouracil rats. This would be expected to enhance the rate of fatty acid oxidation in hyperthyroid rats due to the removal of the inhibition of carnitine palmitoyl transferase 1 . Cettour-Rose and colleagues measured palmitoyl transferase $1 \beta(\mathrm{CPT}-1 \beta)$ expression in muscle, carnitine palmitoyl transferase $1 \alpha(\mathrm{CPT}-1 \alpha)$ expression in white adipose tissue, and muscle triglyceride content in untreated pair-fed and leptin-treated hypothyroid animals [1]. Leptin significantly increased the expression of both muscle CPT- $1 \beta$ and adipose tissue CPT- $1 \alpha$. Leptin also promoted a significant decrease in muscle triglyceride content. Evidence is accumulating for roles of AMPK in controlling glucose uptake, fatty acid oxidation and gene expression in muscle. All subunit isoforms are expressed to a lesser extent in rats treated with propylthiouracil compared with rats given excess thyroid hormones. IRS-1 has been shown to be a target for AMPK [15] and sensitivity of muscle to insulin has recently been found to be influenced by prior AMPK activation [16].

Leptin could be involved in inducing insulin resistance, possibly via peripheral mechanisms of action [17]. Leptin can in fact act through some components of the insulin- signalling cascade, such as IRS-1 and IRS-2, PI 3-kinase and MAPK, and can modify insulin-induced changes in gene expression in vitro and in vivo $[18,19]$. Leptin alone also induces serine phosphorylation of Akt and glycogen synthase 3 , but to a lesser extent than insulin. It rapidly activates signalling pathways directly at the level of insulin-sensitive tissues through the OB-Rb, and these pathways overlap with, but are distinct from, those occupied by insulin [18]. Both leptin-deficient and leptin-resistant obese rodents exhibit severe insulin resistance. This condition is rapidly ameliorated by leptin administration in deficient mice, even before reduction of body weight. Moreover, the insulin-sensitising effect of leptin exceeds that seen in pair-fed animals. Accumulating evidence suggests that leptin promotes fatty acid oxidation and reduces ectopic fat accumulation in non-adipose tissues, thereby increasing insulin sensitivity. The metabolic effects of adipokines are probably mediated by a common pathway involving the activation of AMPK. The activation of AMPK increases beta oxidation and decreases fatty acid esterification to triglycerides, leading to reduced accumulation of lipids in skeletal muscle, adipose tissue and other tissues. Indeed, a role of AMPK in mediating the acute stimulatory effect of leptin on NEFA oxidation was recently demonstrated in murine soleus muscle [20]. Deficiency of leptin (ob/ob mice) or the leptin receptor (Zucker diabetic fatty rat) is associated with a decrease in AMPK activity in muscle and/ or liver, and treatment with an AMPK activator prevents the development of diabetes and ectopic lipid accumulation in the Zucker diabetic fatty rat [21]. Early activation of AMPK occurs by leptin acting directly on muscle, whereas later activation depends on leptin functioning through the hypothalamic-sympathetic nervous system axis. As a result of AMPK activation, the enzyme acetyl coenzyme A carboxylase is inhibited, leading to reduced intracellular levels of the metabolite malonyl CoA. This alleviates inhibition of fatty acid entry into the mitochondria by malonyl CoA and favours fatty acid oxidation. Increased fatty acid oxidation secondary to chronic leptin treatment may be a result of enhanced flux into the mitochondrion through CPT1 regulation. As in the paper presented in this issue by the Geneva group, i.c.v. infusion of leptin places the hormone directly in the central nervous system, where it can stimulate the ventromedial hypothalamus without reaching circulating concentrations that could directly affect peripheral target tissues. Several studies illustrate that prolonged i.c.v. infusion of leptin enhances substrate oxidation so that adipose tissue lipid stores are completely exhausted, and muscle triglyceride and liver glycogen stores depleted, through activation of the sympathetic nervous system and increases in energy expenditure and resting metabolic rate [4]. In this context, thyroid hormones could be important mediators of the effect of leptin on energy expenditure [6]. It is known that i.c.v. leptin regulates BAT via the sympathetic nervous system, and the same tissue is one of the main targets of thyroid hormones. Moreover, the activity of BAT may indirectly influence both peripheral glucose and lipid metabolism in rats. 
The role of the thyroid-leptin axis in the homeostasis of glucose, lipid and energy balance is becoming clearer. Based on the available evidence from molecular biology, the pivotal regulatory role of $\mathrm{T} 3$ in major metabolic pathways has been delineated by mapping the specific action sites of T3 and leptin on the metabolic pathways of the glucose-lipid cycle. The paper in this issue of Diabetologia presents an integrative hypothesis of the synergistic relationship of T3 and leptin in metabolic homeostasis [1]. Hypothyroidism is characterised by decreased insulin responsiveness, partly mediated by an exaggerated glucosefatty acid cycle that is improved by i.c.v. leptin administration.

\section{References}

1. Cettour-Rose P, Theander-Carrillo C, Asensio C et al (2005) Hypothyroidism in rats decreases peripheral glucose utilisation, a defect partially corrected by central leptin infusion. Diabetologia 48. DOI 10.1007/s00125-005-1696-4

2. Magnus-Levy A (1895) Ueber den respiratorischen Gaswechsel unter Einfluss de Thyroidea sowie unter verschiedenen pathologische Zustand. (Article in German). Berlin Klin Wochschr 32:650-652

3. Yen PM (2001) Physiological and molecular basis of thyroid hormone action. Physiol Rev 81:1097-1142

4. Wang JL, Chinookoswong N, Yin S, Shi ZQ (2000) Calorigenic actions of leptin are additive to, but not dependent on, those of thyroid hormones. Am J Physiol Endocrinol Metab 279:E1278E1285

5. Escobar-Morreale HF, Escobar del Rey F, Morreale de Escobar G (1997) Thyroid hormones influence serum leptin concentrations in the rat. Endocrinology 138:4485-4488

6. Cusin I, Rouru J, Visser T, Burger AG, Rohner-Jeanrenaud F (2000) Involvement of thyroid hormones in the effect of intracerebroventricular leptin infusion on uncoupling protein-3 expression in rat muscle. Diabetes 49:1101-1105

7. Elmquist JK (2001) Hypothalamic pathways underlying the endocrine, autonomic, and behavioral effects of leptin. Physiol Behav 74:703-708

8. Harris M, Aschkenasi C, Elias CF et al (2001) Transcriptional regulation of the thyrotropin-releasing hormone gene by leptin and melanocortin signalling. J Clin Invest 107:111-120
9. Seoane LM, Carro E, Tovar S, Casanueva FF, Dieguez C (2000) Regulation of in vivo TSH secretion by leptin. Regul Pept 92:25-29

10. Kohrle J (1999) Local activation and inactivation of thyroid hormones: the deiodinase family. Mol Cell Endocrinol 151: 103-119

11. Dimitriadis GD, Leighton B, Parry-Billings M, West D, Newsholme EA (1989) Effects of hypothyroidism on the sensitivity of glycolysis and glycogen synthesis to insulin in the soleus muscle of the rat. Biochem J 257:369-373

12. Weinstein SP, Watts J, Haber RS (1991) Thyroid hormone increases muscle fat glucose transporter gene expression in rat skeletal muscle. Endocrinology 129:455-464

13. Casla A, Rovira A, Wells JA, Dohm GL (1990) Increased glucose transporter (GLUT4) protein expression in hyperthyroidism. Biochem Biophys Res Commun 171:182-188

14. Winder WW, Holmes BF, Rubink DS, Jensen EB, Chen M, Holloszy JO (2000) Activation of AMP-activated protein kinase increases mitochondrial enzymes in skeletal muscle. J Appl Physiol 88:2219-2226

15. Jakobsen SN, Hardie DG, Morrice N, Tornqvist HE (2001) 5'AMP-activated protein kinase phosphorylates IRS-1 on Ser-789 in mouse $\mathrm{C} 2 \mathrm{C} 12$ myotubes in response to 5-aminoimidazole-4carboxamide riboside. J Biol Chem 276:46912-46916

16. Fisher JS, Gao J, Han DH, Holloszy JO, Nolte LA (2002) Activation of AMP kinase enhances sensitivity of muscle glucose transport to insulin. Am J Physiol Endocrinol Metab 282: E18-E23

17. Frühbeck G, Jebb SA, Prentice AM (1998) Leptin: physiology and pathophysiology. Clin Physiol 18:399-419

18. Kim YB, Uotani S, Pierroz DD, Flier JS, Kahn BB (2000) In vivo administration of leptin activates signal transduction directly in insulin-sensitive tissues: overlapping but distinct pathways from insulin. Endocrinology 141:2328-2339

19. Szanto I, Kahn CR (2000) Selective interaction between leptin and insulin signaling pathways in a hepatic cell line. Proc Natl Acad Sci U S A 97:2355-2360

20. Minokoshi Y, Kim YB, Peroni OD, Fryer LG, Muller C, Carling D, Kahn BB (2002) Leptin stimulates fatty-acid oxidation by activating AMP-activated protein kinase. Nature 415:339-343

21. Yu X, McCorkle S, Wang M et al (2004) Leptinomimetic effects of the AMP kinase activator AICAR in leptin-resistant rats: prevention of diabetes and ectopic lipid deposition. Diabetologia 47:2012-2021 